\title{
Quantificação dos Recursos Eólicos no Estado do Piauí através de simulações computacionais
}

Quantification of Wind Resources in the State of Piaui through computer simulations

\section{Marcos Antonio Tavares Lira, José Machado Moita Neto, Emerson Mariano da Silva e Bruno Pires Sombra}

\author{
${ }^{1}$ Mestre, Departamento de Engenharia Elétrica, Universidade Federal do Piauí, Teresina, Brasil \\ marcoslira@ufpi.edu.br \\ ${ }^{2}$ Departamento de Engenharia Elétrica, Universidade Federal do Piauí, Teresina, Brasil \\ jmoita@ufpi.edu.br \\ ${ }^{3}$ Doutor, Departamento de Física, Universidade Estadual do Ceará, Fortaleza, Brasil \\ emerson.mariano@uece.br \\ ${ }^{4}$ Mestre, Departamento de Física, Instituto Federal de Educação, Ciência e Tecnologia do Piauí, Parnaíba, \\ Brasil \\ brunosombra@hotmail.com
}

\begin{abstract}
Resumo
A energia eólica têm-se destacado no contexto mundial por ser uma fonte de energia limpa e uma alternativa ao uso das fontes convencionais. Como a variabilidade da velocidade e direção do vento depende das condições climáticas, o desenvolvimento de ferramentas que possam estimar estas grandezas tem papel singular. Dessa forma, esse trabalho tem por objetivo estimar o perfil eólico do Estado do Piauí por meio de simulações computacionais através do modelo atmosférico BRAMS. Foi possível ainda destacar o campo vetorial do vento e as zonas de maior potencial eólico através de mapas gerados por computador
\end{abstract}

Palavras-chave: Modelagem, energia eólica, estimativa de ventos.

\begin{abstract}
Wind energy has been prominent in the global context to be a clean energy source and an alternative to the use of conventional sources. As the variability of wind speed and direction depends on climatic conditions, the development of tools that can estimate these quantities have singular role. Thus, this study aims to estimate the wind profile of the state of Piaui through computer simulations using the atmospheric model BRAMS. It was also possible to highlight the wind vector field and the areas of greatest wind potential through computer generated maps.
\end{abstract}

Keywords: Modeling, power wind, estimated winds. 


\section{Introdução}

A partir da década de 70 diversas transformações sociais e ambientais tornaram evidente a necessidade de novas matrizes energéticas. Essa necessidade ficou ainda mais clara com a crise do petróleo de 1973 e 1979, quando se percebeu a limitação das fontes de energias convencionais como o petróleo, o carvão, o gás natural ou a energia nuclear, as quais em maior ou menor escala impactam o meio ambiente (RÍO \& BURGUILLO, 2008). Assegurar o acesso a fontes de energia limpas e sustentáveis é, sem dúvida, um dos grandes desafios enfrentados pelo mundo moderno (DOBRIANSKY, 2006).

A utilização do vento como recurso para a produção de energia elétrica teve grande impulso na década de 90 através de vários programas executados em vários países que motivaram investimentos em pesquisas e desenvolvimento. Com o aumento do número de fabricantes de turbinas eólicas, estas tiveram sua performance melhorada e seus custos reduzidos (CUSTÓDIO, 2013).

A energia eólica tem se destacado como a fonte de energia que vem se expandindo com maior velocidade em escala mundial, superando fontes convencionais como o gás natural e a energia nuclear. A Europa continua sendo a região protagonista em produção de energia eólica e esta posição tende a se consolidar cada vez mais, uma vez que vários de seus países tem investido neste tipo de geração (LÓPEZ, 2011).

Na Europa, a expansão do uso da energia eólica tem se notabilizado não apenas na Alemanha e Espanha, mas também Dinamarca e Reino Unido passaram a ser produtores importantes dessa fonte (CASTRO, 2007). Segundo a Associação Brasileira de Energia Eólica (ABEEólica), o Brasil iniciou 2015 com 241 usinas eólica instaladas, totalizando $6 \mathrm{GW}$ de capacidade instalada após o início de testes de quatro novos parques na Bahia (Ametista de 28,54 MW, Maron de 30,24 MW, Pilões de 30,24 MW e Dourados 28,58 MW).

A energia eólica passar a responder por $4,5 \%$ da matriz elétrica nacional. De acordo com a ABEEólica, o crescimento deve continuar, mantendo a projeção da entidade de terminar o ano de 2015 com 9,8 GW de capacidade instalada. Este cálculo é baseado nos projetos contratados nos últimos leilões. Com a entrada em operação de novos parques nos próximos anos, o Brasil alcançará uma potencia instalada de 15,2 GW em 2019.

O Piauí é um estado com potencial para a geração de até 1000 MW em energia eólica. Atualmente, são produzidos no estado apenas 88 megawatts (MW) oriundos de 2 usinas eólicas instaladas no litoral piauiense. Até julho de 2015 a produção de energia eólica no Piauí deve chegar a 400 MW e para 2016 a expectativa é de que o estado alcance uma capacidade instalada de $560 \mathrm{MW}$.

A energia eólica também pode produzir impactos ambientais, apesar de não estarem relacionadas à emissão de gases ou resíduos. Em geral, os benefícios ambientais da energia eólica estimados pelas emissões evitadas de outras fontes (PINTO, 2013).

Segundo Martin (2008), as maiores fontes de incertezas estão relacionadas à modelagem dos recursos eólicos representativos para os anos subsequentes ao início de operação do parque. Desta forma é feita à predição futura e em longo prazo do comportamento do vento, a partir da extrapolação dos recursos eólicos coletados no local do projeto durante poucos meses ou anos, os quais não apresentam representatividade climatológica.

No Brasil, e especificamente na Região Semiárida do Nordeste (RSANEB), diferentemente da realidade observada na Europa, não se encontra um grande número de estações meteorológicas de superfície próximas aos parques eólicos instalados, o que de antemão já inviabiliza o uso dos procedimentos avançados de correlação utilizados naquela região. Sendo assim, uma alternativa para resolução desse problema, nessa região, seria o uso dos dados de previsões numéricas de velocidade e direção do vento.

Dessa forma, através de um maior potencial de processamento, esta pesquisa pretende desenvolver procedimentos metodológicos próprios, visando a quantificação de recursos eólicos, com maior precisão, com auxílio de modelagem numérica, nas regiões litorâneas e continentais da RSANEB, e particularmente do Estado do Piauí. 


\section{Metodologia}

O Brazilian developments on the Regional Atmospheric Modeling System (BRAMS) é um modelo computacional de previsão através de simulação numérica altamente versátil, desenvolvido e atualizado por vários grupos, inclusive brasileiros.

O modelo se baseia nas equações que governam o movimento das massas de ar, notadamente a definição tridimensional de vento (eq. 1), sua magnitude (eq. 2) e a equação da continuidade (eq. 3) para conservação da massa.

$$
\begin{aligned}
& \vec{V}=u \hat{i}+v \hat{j}+w \hat{k} \\
& \|\vec{V}\|=\sqrt{u^{2}+v^{2}+w^{2}} \\
& \frac{\partial \rho}{\partial t}+\nabla \cdot(\rho \vec{u})=0
\end{aligned}
$$

em que:

$\vec{V}$ é o vetor velocidade do vento;

u, v e w são as componentes do vetor $\vec{V}$;

$\square$ é a massa específica do ar.

Para iniciar o processamento, o modelo faz uso das condições iniciais e de contorno que envolvem as características da superfície, tais como temperatura de superfície do mar (TSM), topografia, vegetação, uso do solo e classes de textura do solo. A precisão do modelo é fortemente influenciada pelas grades de interpolação. Uma grade é uma malha que define os pontos de interpolação do modelo. Quanto mais pontos de interpolação, maior resolução o modelo terá para realizar a simulação da região em questão.

Para execução do BRAMS, pode-se optar por diferentes tamanhos de grades. Deste modo, a mudança de grade para uma resolução maior ou menor pode influenciar os resultados, tornandoo mais ou menos representativo para o evento, como por exemplo: microescala (Camada Limite Atmosférica), mesoescala (Brisa Marítima) e Sinótica (Frente Fria).

O tempo de processamento cresce com o aumento da resolução e do domínio. O tempo de processamento deve obedecer à condição Courant Friederichs Lewy (CFL), que consiste numa propriedade que usa aproximação de diferenças finitas, de modo que, se houver violação desta condição, ocorrerá uma instabilidade numérica ocasionando um erro durante a execução do modelo.

Com a finalidade de aumentar a eficiência do modelo, pode-se aninhar diferentes grades com diferentes resoluções e obter um melhor refinamento para a área de interesse. A partir dos dados de reanálises obtida no site do Centro de Previsão de Tempo e Estudos Climáticos/Instituto Nacional de Pesquisas Espaciais (CPTEC/INPE), realizou-se a configuração e parametrização do modelo BRAMS através dos arquivos RAMSIN, os quais são arquivos disponibilizados pelo BRAMS para configuração da simulação.

Foram realizadas diversas simulações numéricas com o modelo BRAMS a fim de definir a dinâmica da atmosfera para a região delimitada, a qual é mostrada na Figura 1. O modelo foi alimentado com dados de medição das variáveis que influenciam a dinâmica da atmosfera, fornecidos pelo CPTEC/INPE, sobretudo velocidade e direção do vento.

Ao fim da simulação, que levou cerca de 6 (seis) horas, o modelo gerou alguns arquivos de saída para análises, obtidos na altitude de 14,5 metros, os quais foram utilizados para gerar imagens que descrevem o cenário da região em questão (Figura 1), em termos de magnitude e direção do vento.

As simulações foram realizadas com dados da última semana de julho, o mês de agosto inteiro e a primeira semana de setembro do ano de 2012. O modelo utilizado pelo BRAMS é tão complexo e preciso que ele consegue por meio desses pontos de medição estimar (prever) as condições da atmosfera para o local simulado, extrapolando as medições finitas realizadas em pontos discretos.

Neste estudo, embora a região simulada tenha sido o estado do Piauí, a área de simulação foi um pouco maior que o estado, para que o modelo pudesse levar em conta as influências da atmosfera no entorno da região de interesse. Da mesma forma, como o mês de interesse foi agosto, a simulação se iniciou na última semana de julho e parte de setembro, para também levar em conta a influência da atmosfera em períodos anteriores e posteriores. 


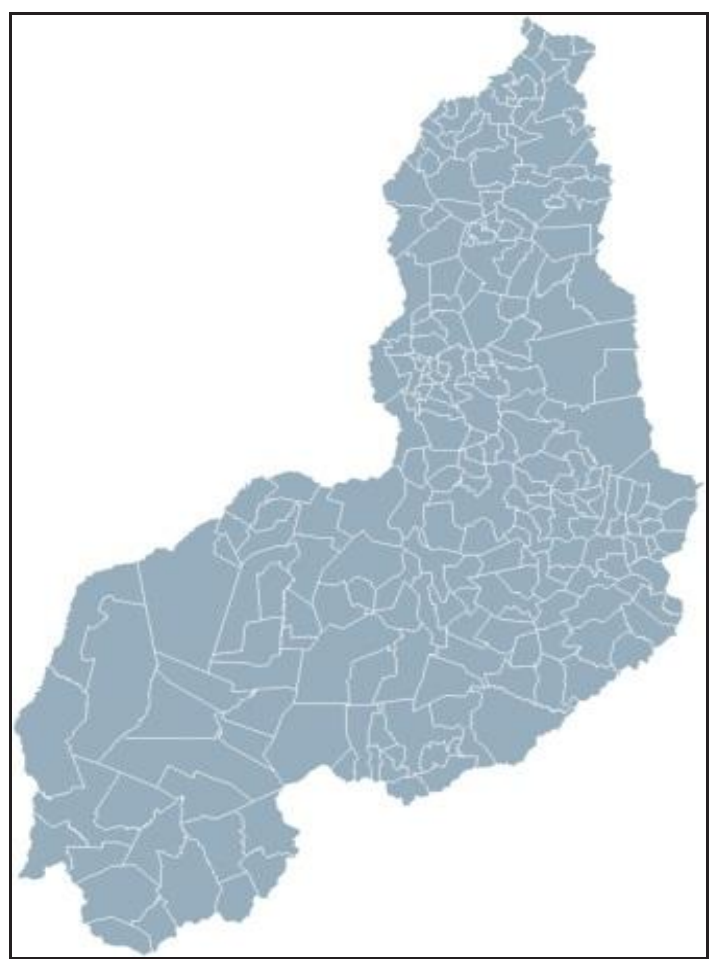

Figura 1: Região simulada com o BRAMS.

Fonte:http://www.uep.cnps.embrapa.br/solos/m apas/piaui.jpg.

\section{Resultados e discussões}

Em princípio, pelo resultado da simulação, pode-se verificar o grande potencial eólico presente na extensa região Sudeste do Piauí, onde verificam-se valores médios de velocidade do vento acima de $12 \mathrm{~m} / \mathrm{s}$, como mostram as Figuras 2 e 3. Estes resultados caracterizam-se como parâmetros para a estimativa da potência que pode ser extraída do vento por metro quadrado de área varrida pelas pás dos aerogeradores (densidade de potência). Pela Tabela 1 é possível estimar quantitativamente a potência que pode ser convertida em energia elétrica. Deve ser levado em conta também o fato de que as turbinas oferecem resistência a esta conversão, logo há pequenas perdas.

A região que se mostra mais favorável ao aproveitamento de energia eólica compreende a parte piauiense da Chapada do Araripe. O principal fator que contribui para estes índices é a altitude, a qual pode chegar a 750 metros em alguns municípios (Marcolândia, Caldeirão Grande). Estes resultados confirmam a razão pela qual os novos empreendimentos eólicos estão se instalando nesta região do estado. Este caráter favorável ao aproveitamento do vento em regiões de elevadas altitudes já foi comprovado em estudos anteriores (CARVALHO et al., 2012; RAMOS et al., 2013; TEIXEIRA \& CAMELO, 2013).

Tabela 1: Estimativas da densidade de potência do vento em função da velocidade média do vento.

\begin{tabular}{c|c}
\hline $\begin{array}{c}\text { Velocidade do Vento } \\
(\mathbf{m} / \mathbf{s})\end{array}$ & $\begin{array}{c}\text { Densidade de } \\
\text { Potência }\left(\mathbf{W} / \mathbf{m}^{2}\right)\end{array}$ \\
\hline 0 & 0 \\
\hline 1 & 1 \\
\hline 2 & 5 \\
\hline 3 & 17 \\
\hline 4 & 39 \\
\hline 5 & 77 \\
\hline 6 & 132 \\
\hline 7 & 210 \\
\hline 8 & 314 \\
\hline 9 & 447 \\
\hline 10 & 613 \\
\hline 11 & 815 \\
\hline 12 & 1058 \\
\hline 13 & 1346 \\
\hline 14 & 1681 \\
\hline 15 & 2067 \\
\hline
\end{tabular}

Fonte: Masters, 2004.
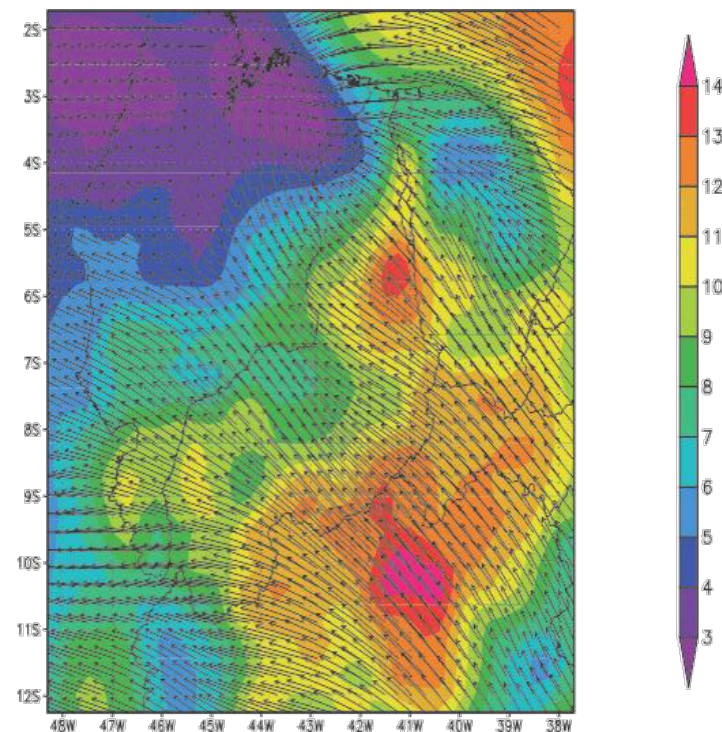

Figura 2: Magnitude da velocidade média do vento e direção predominante em alta resolução. 


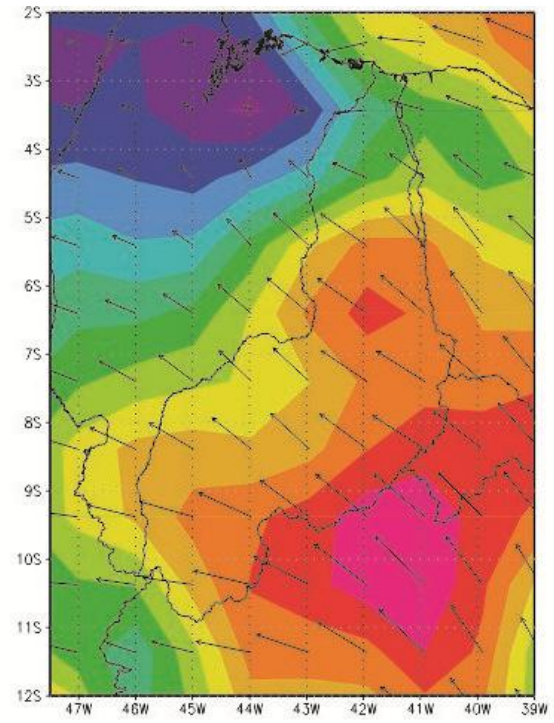

Figura 3: Magnitude da velocidade média do vento e direção predominante em resolução menor.
Observa-se nas figuras acima que a direção predominante do vento na região simulada é de sudeste, com algumas variações na direção leste evidenciando a influência dos Ventos Alísios na região, conforme estudos realizados por Barreto, Aragão e Braga (2002).

Após a simulação, escolhemos dois municípios piauienses (Itainópolis e Parnaíba) para compararmos os seus respectivos valores de velocidade (medidos) com os valores apresentados nas simulações. As figuras 4 e 5, respectivamente, mostram os perfis de velocidade média dos ventos dos municípios de Itainópolis e Parnaíba ao longo do mês de agosto de 2012.

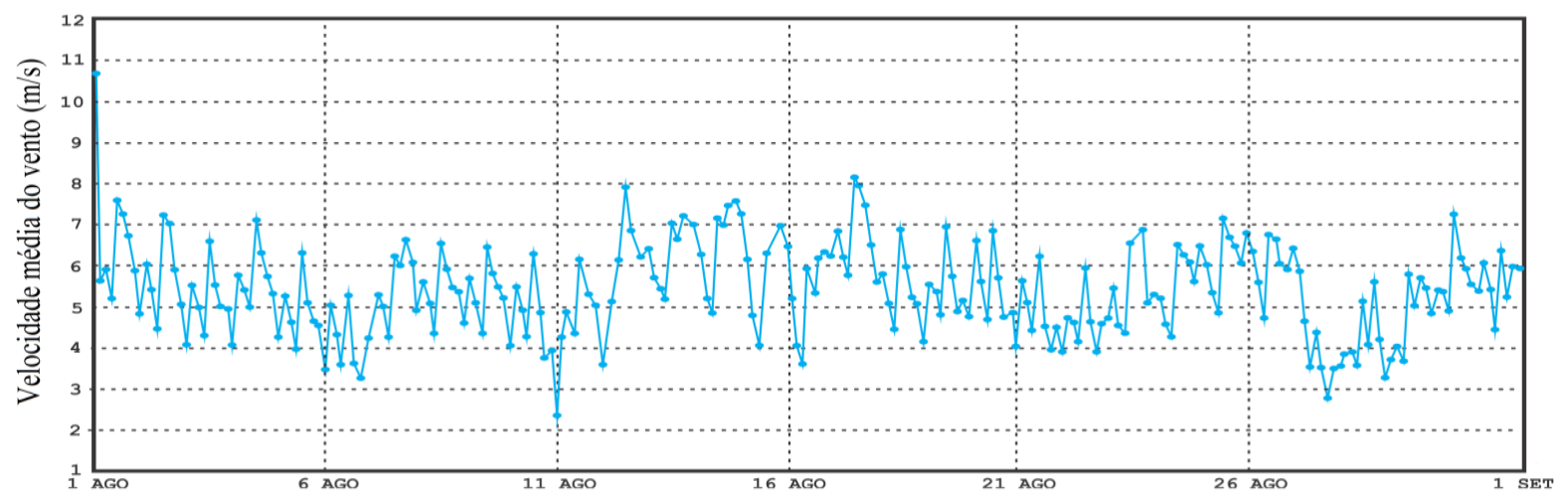

Figura 4: Perfil da velocidade do vento no município de Itainópolis no mês de agosto/2012.

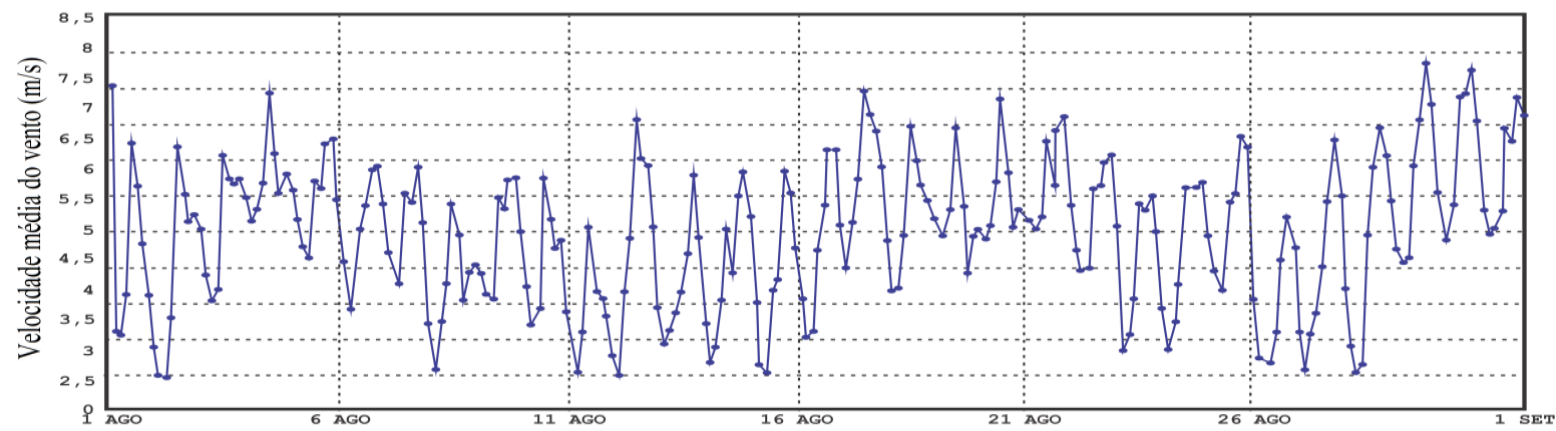

Figura 5: Perfil da velocidade do vento no município de Parnaíba no mês de agosto/2012.

Pelo perfil de velocidade do vento da cidade Itainópolis, a maior parte dos valores de velocidade do vento estão acima dos $5 \mathrm{~m} / \mathrm{s}$, com velocidade máxima acima de $8 \mathrm{~m} / \mathrm{s}$. Pela simulação, o município aparece na região onde a velocidade está em torno de $10 \mathrm{~m} / \mathrm{s}$. Como os valores mostrados na simulação são uma média dos valores ao longo de todo o mês de agosto, este resultado pode ser visto como uma aproximação razoável. Já para o perfil de velocidade média de Parnaíba, a maioria dos valores medidos estão acima de $4 \mathrm{~m} / \mathrm{s}$, com velocidade de pico em torno de $8 \mathrm{~m} / \mathrm{s}$. Pelo resultado da simulação este município aparece no mapa com velocidades de vento na faixa de $6 \mathrm{~m} / \mathrm{s}$ a $7 \mathrm{~m} / \mathrm{s}$. 
Alguns aerogeradores, como é o caso do modelo E 701.8 da fabricante Wobben, possuem em sua curva de potência, uma velocidade de entrada - velocidade na qual se tem o início da geração - de 2,5 m/s. Levando-se em consideração esta informação, observa-se que, em ambos os municípios, quase que a totalidade dos valores de velocidade são maiores ou iguais à velocidade de entrada citada. Em ambos os casos evidencia-se que estas regiões em potencial para o aproveitamento de recursos eólicos na perspectiva de produção de energia elétrica.

\section{Conclusões}

De acordo com os resultados apresentados com uso do modelo BRAMS, a simulação computacional demonstrou ser uma ferramenta viável ao estudo e análise de potenciais parques eólicos, uma vez que, na medida em que se baseia nas equações que governam a dinâmica da atmosfera, produz dados de saída com um grau elevado de aproximação entre os dados medidos e os simulados.

O estudo mostrou que embora a região costeira seja notadamente favorável ao aproveitamento de energia eólica, a região serrana também aparece como propícia devido ao aspecto da altitude elevada. Tanto o estudo de viabilidade quanto o projeto e definição dos equipamentos utilizados para montagem de usinas podem se utilizar do presente trabalho para seus objetivos, uma vez que a simulação computacional, além de ser economicamente viável, produz resultados satisfatórios, próximos dos valores medidos.

A simulação foi realiza somente para o mês de agosto, no entanto para trabalhos futuros sugerimos a simulação anual. Para tanto faz-se necessário contar com computadores com elevada capacidade de processamento e memória, uma vez que a simulação pode lavar dias dada a enorme quantidade de dados a serem simulados.

\section{Referências}

Alcântara, C. R.; SOUZA, E. P. Uma teoria termodinâmica para brisas: testes utilizando simulações numéricas. Rev. Bras. Meteor. v.23, n.1, p.1-11, março, 2008.
Almeida, G.P. ; Leal Júnior, J. B. V.; Pelegrinni, C.C.; Lyra, R. F. F.; Fisch, G. F. Simulação da circulação de brisa marítima e terrestre durante $o$ experimento CHUVA em Fortaleza. Ciência e Natura, v. Suplem, p. 267-269, 2011.

Barrreto, A. B.; Aragão, M. R. S.; Braga, C. C. Estudo do ciclo diário do vento à superfície no Nordeste do Brasil. In: CONGRESSO BRASILEIRO DE METEOROLOGIA, 12, 2002, Foz de Iguaçu. Anais XII Congresso Brasileiro de Meteorologia, 2002.

Carvalho, I. V.; Mesquita, A. L.; Santos, P. G. de L.; Santos, J. S.. Densidade de potencial eólico nas diferentes regiões do estado do Ceará litoral, serra, sertão. Revista Geonorte, Edição Especial 2, V.2, N.5, p.1317 - 1327, 2012.

Carvalho, P. Geração eólica. Fortaleza: Imprensa Universitária, 2003. 146 p.

Castro, R. M. G. Energias Renováveis e Produção Descentralizada: introdução à energia eólica. 3 ed. Lisboa, Universidade Técnica de Lisboa, 2007.

Custódio, R. Energia Eólica. São Paulo: Eletrobrás, 2013.

Dobriansky, P. Energia limpa para o futuro. Revista eJournal USA: Perspectivas Econômicas, Washington, D.C, v 11, n. 2, 4-6, 2006.

Global Wind Energy Council. Global Wind Report - Annual market update 2011 (2011). Relatório Anual de Energia Eólica.

López, J. M. E. Manual de Energia Eólica. 2 ed. Madrid: Ediciones Mundi-Prensa, 2011.

Martin, F. R.; Guarnieri, R. A.; Pereira, E. B. O aproveitamento da energia eólica. Revista Brasileira de Ensino de Física, São Paulo, v. 30, n. 1, março, 2008.

Masters, G. M. Renewable and eficiente electric power systems. New Jersey: John Wiley and Sons, 2004.

Pinto, M. de O. Fundamentos da Energia Eólica. Rio de Janeiro, LTC, 2013.

Ramos, D. N. da S.; Lyra, R. F. da F.; Silva Júnior, R. S. Previsão do vento utilizando o modelo atmosférico WRF para o estado de Alagoas. Rev. Bras. Meteor., São Paulo, vol.28, n.2, Junho, 2013. 
Río, P.; Burguillo, M. Assessing the impact of renewable energy deployment on local sustainability: Towards a theoretical framework. Renewable and Sustainable Energy Reviews, v.12, n.5, p.1325-44, jun. 2008.

Santiago de Maria, P. H. S.; Costa, A. A.; Sombra, S. S. Simulação de ventos em alta resolução no litoral do Ceará. In: CONGRESSO BRASILEIRO DE METEOROLOGIA, 14, 2006, Florianópolis. Florianópolis. Anais...XIV Congresso Bras. de Meteorologia, 2006.

Stull, R.B. An introduction to Boundary Layer Meteorology. (Kluwer Academic Publishers, Dordrecht, 1988).

Teixeira, W. F.; Camelo, H. do N. Análise da densidade de potência eólica em regiões de serra e litoral do estado do Ceará. Revista Ciência e Natura, Santa Maria, Edição Esp. Dez. 2013. p. $396-399$. 